\title{
A Yaw Stability Control Algorithm for Four-Wheel Independently Actuated Electric Ground Vehicles considering Control Boundaries
}

\author{
Cheng Lin, ${ }^{1}$ Zhifeng Xu, ${ }^{1}$ and Ru Zhang ${ }^{2}$ \\ ${ }^{1}$ National Engineering Laboratory for Electric Vehicle, Beijing Institute of Technology, Beijing 100081, China \\ ${ }^{2}$ Pan Asia Technical Automotive Center, Shanghai 201201, China \\ Correspondence should be addressed to Zhifeng Xu; xzf2012@126.com
}

Received 4 June 2014; Accepted 15 August 2014

Academic Editor: Yuming Qin

Copyright (C) 2015 Cheng Lin et al. This is an open access article distributed under the Creative Commons Attribution License, which permits unrestricted use, distribution, and reproduction in any medium, provided the original work is properly cited.

\begin{abstract}
A hierarchical control algorithm of direct yaw moment control for four-wheel independently actuated (FWIA) electric ground vehicles is presented. Sliding mode control is adopted to yield the desired yaw moment in the higher layer of the algorithm due to the possible modeling inaccuracies and parametric uncertainties. The conditional integrator approach is employed to overcome the chattering issue, which enables a smooth transition to a proportional + integral-like controller, with antiwindup, when the system is entering the boundary layer. The lower level of the algorithm is given to allocate the desired yaw moment to four wheels by means of slip ratio distribution and control for a better grasp of control boundaries. Simulation results, obtained with a vehicle dynamics simulator, Carsim, and the Matlab/Simulink, show the effectiveness of the control algorithm.
\end{abstract}

\section{Introduction}

Electric vehicles are more environmentally friendly and energy efficient than internal combustion engine vehicles (ICEVs). The electrification of vehicles is the effective way to solve environmental and energy issues. Moreover, as the driving torque of motors can be regulated accurately, electric vehicles also have advantages over ICEVs in terms of applying driving torque regulation-based dynamic control algorithms, such as slip ratio control. Yaw stability is significant for vehicle safety. As driving/braking torque of each wheel can be controlled accurately, FWIA electric vehicles are more convenient for differential driving/braking, which has enriched means of direct yaw moment control. Compared with other stability control systems, differential driving-/braking-based systems are more effective in vehicle yaw stability control especially when tire is working in the nonlinear area [1]. However, differential braking will reduce vehicle longitudinal velocity dramatically, which is not expected by the driver. The combination of differential driving and differential braking can prevent unexpected change in vehicle speed during yaw stability control.
Approaches developed by researchers for vehicle yaw stability control usually consist of a yaw moment calculation layer and a wheel torque distribution and control layer. Sliding mode control (SMC) [2], fuzzy logic control [3], feed forward and feedback control [4], $H_{\infty}$ robust control [5], model predictive control [6], and adaptive control [7] all have been used in calculating the desired yaw moment. In terms of the wheel torque distribution and control layer, typical methods introduced in the available literatures are shown as follows. In [8], the authors calculated the desired longitudinal and lateral tire forces based on feedback linearization algorithm; adhesion ellipse was used to prescribe a limit to those two forces. Then the desired wheel slip ratio and slip angle were calculated from the inverse tire model. But without active steering system, it may be difficult to realize the desired longitudinal and lateral forces simultaneously. In [9], the authors allocated each tire's target slip ratio and slip angle from desired forces in the longitudinal, lateral, and yaw directions by solving quadratic programming problems; then the target tire slip and slip angle were tracked based on ABS/ ASR/AWS. Quadratic programming was used in the control 


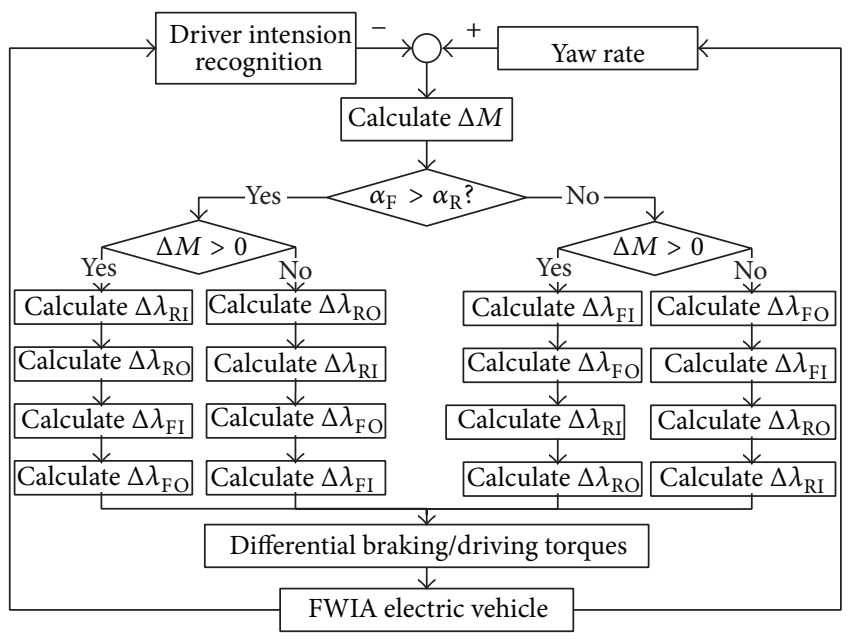

FIGURE 1: Structure of the proposed algorithm.

allocation which may have high computational cost. In [10], the authors developed a longitudinal tire force distribution strategy called "LoFDDS" in which wheels' braking/driving torques were controlled within rules; tires' adhesion status was detected at real time to observe whether they are at risk or not. In addition, some papers have proposed torque distribution methods for drive condition with both acceleration and turning. In [11], a proportional-integral control strategy was implemented, applying yaw rate feedback to vary the front-rear torque distribution and lateral acceleration feedback to adjust the left-right distribution. In [12], the authors developed a wheel torque regulation strategy based on fuzzy logic control; the yaw rate error and its rate of change were used as the controller inputs. As the wheel torques can be accurately controlled on the FWIA electric vehicles, finding a method that can accurately control the vehicle yaw motion with lower computational cost is meaningful.

The main contributions of this study are that a hierarchical algorithm of yaw stability control for FWIA electric vehicles is presented. A SMC with conditional integrator (SMC\&CI) based higher-layer controller is used to yield the desired yaw moment, which not only keeps the advantages of SMC, but also eliminates jitter around the sliding surface with a higher tracking accuracy. In the second layer of the proposed algorithm, unlike what the authors did in literature mentioned above, the desired yaw moment is allocated to wheel slip ratio directly instead of tire longitudinal and lateral forces, according to a preset order which is helpful to inhibit the oversteer and understeer trend of the vehicle. And the allocation process has no online optimization part which may have heavy computational cost. Boundaries of each slip ratio are calculated to avoid vehicle entering dangerous zone of adhesion.

The remainder of the paper is structured as follows. The overall structure of the proposed direct yaw moment control algorithm and the process of yaw moment calculation are introduced in Section 2. Section 3 provides the introduction of the wheel torque distribution and control layer. Section 4

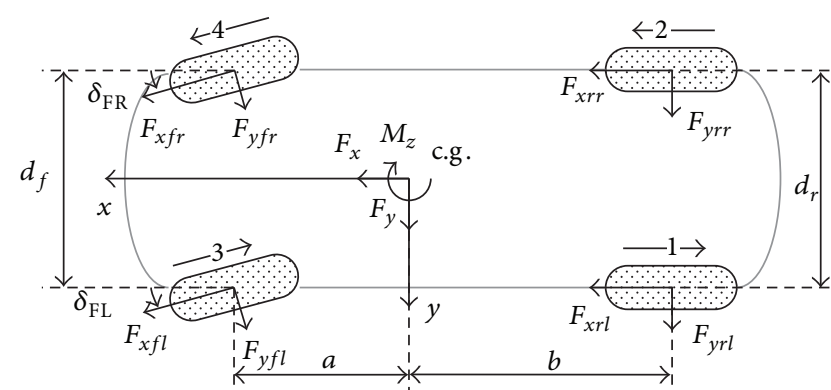

FIGURE 2: Schematic diagram of yaw stability control.

shows the simulation results and related discussions. At last, the conclusions are given in Section 5.

\section{The Hierarchical Direct Yaw Control Algorithm}

2.1. Structure of the Proposed Algorithm. The overall structure of the proposed algorithm is shown in Figure 1, where $\Delta M$ is the desired yaw moment, $\alpha_{\mathrm{F}}$ and $\alpha_{\mathrm{R}}$ are the side slip angle of the front and rear axle, respectively, and $\Delta \lambda_{\mathrm{FI}}, \Delta \lambda_{\mathrm{FO}}, \Delta \lambda_{\mathrm{RI}}$, and $\Delta \lambda_{\mathrm{RO}}$ are the desired slip ratio of the front inside wheel, front outside wheel, rear inside wheel, and rear outside wheel, respectively.

A model suggested in [13] is used as the desired yaw rate reference model. The difference between the desired yaw rate and observed value is used to calculate the desired yaw moment based on SMC\&CI. The lateral adhesion status of the axles is evaluated and the better one is chosen as a priority to generate the desired yaw moment. After that, the desired slip ratios of wheels on the prior axle are calculated according to the desired yaw moment. One thing to note here is that braking one wheel takes precedence over driving one. This can avoid the sudden increase in vehicle speed which may deteriorate the adhesion state. If braking of one wheel on the priority axle cannot generate enough yaw moment, driving torque will be applied to the other wheel of this axle. On condition that yaw moment generated still cannot meet the demand, braking/driving torque will be applied to wheels on the other axle.

Take the left turning vehicle with understeer trend shown in Figure 2 as an example. After getting the desired yaw moment, the braking slip ratio of rear left wheel is calculated and then the driving slip ratio of the rear right wheel. This is because on condition of understeer side slip angle of the rear axle is smaller than that of the front axle [14]. Increase in the tire longitudinal force may result in decrease in tire lateral stiffness and enlargement in axle side slip angle when vehicle is cornering. Therefore, rear axle is chosen to be the priority and yaw control task is primarily allocated on this axle for balance. As shown in Figure 2, the vehicle is controlled in the following order: applying braking torque on the rear left wheel, applying driving torque on the rear right wheel, applying braking torque on the front left wheel, and then driving torque on the front right wheel if needed. In the end, slip ratio is controlled using methods described in [15]. 


\subsection{Higher-Layer Controller Design}

2.2.1. SMC Based Controller. SMC is used to yield the vehicle virtual control effort due to the possible modeling inaccuracies and parametric uncertainties.

The vehicle stress state is shown in Figure 2. Around the $z$-axis of the vehicle coordinate system, there is

$$
M_{0}(\Omega)-\Delta M=-I_{z} \dot{\Omega},
$$

where $M_{0}(\Omega)$ is the moment around $z$-axis from road, $\Delta M$ is the needed yaw moment based on $M_{0}(\Omega), I_{z}$ is the vehicle moment of inertia around $z$-axis, and $\Omega$ is the vehicle yaw rate.

In the SMC framework adopted in this paper, the control objective is to reach and remain in sliding surface $e=0$, where

$$
e=\Omega-\Omega_{d}
$$

$\Omega_{d}$ is the desired yaw rate by the driver. And first-order asymptotically stable desired error dynamics is defined as follows:

$$
\dot{e}=-k \operatorname{sgn}(e) \text {. }
$$

The sliding control law is defined as

$$
\Delta M=\widehat{M}_{0}(\Omega)-I_{z} k \operatorname{sgn}(e)
$$

where $\widehat{M}_{0}(\Omega)$ is the estimate of $M_{0}(\Omega)$.

The SMC algorithm has some attractive properties in terms of the performance and robustness. However, there are some practical issues related to the chattering which pose practical obstacles to its implementation. These problems are induced by the discontinuous control law, which can cause undesirable high-frequency oscillations in the control signal, excite nonmodeled dynamics, produce noise and wear in the mechanical system, and so forth.

To overcome the problem above, the general approach is to introduce a continuous approximation

$$
\operatorname{sat}\left(\frac{e}{\varepsilon}\right)=\operatorname{sgn}\left(\frac{e}{\varepsilon}\right) \min \left(1,\left|\frac{e}{\varepsilon}\right|\right)
$$

to replace the discontinuous function $\operatorname{sgn}(\cdot)$. Then we get a continuous sliding control law as follows:

$$
\Delta M=\widehat{M}_{0}(\Omega)-I_{z} k \operatorname{sat}\left(\frac{e}{\varepsilon}\right),
$$

where $\varepsilon>0$ is the width of the boundary layer.

Equation (6) gives the needed yaw moment around vehicle $z$-axis, where saturation function is used to mitigate jitter. But there will be steady-state tracking error [16]. To alleviate this problem, a conditional integrator will be introduced to the sliding control law.

2.2.2. Application of the Conditional Integrator. In order to address the steady-state error, an integral action can be introduced to SMC. The application of integral control in the SMC can ensure the asymptotic regulation in the presence of constant disturbances. Moreover, the transient performance of SMC will be degraded and special care must be taken to avoid integrator windup. The conditional integrator in [17] is introduced with the SMC in this paper, which allows us to keep the performance of idea SMC outside the boundary layer and switch to a PI-like controller, with antiwindup mechanism when the yaw rate is in the proximity of the setpoint.

To build such a controller, a new term-conditional integrator will be added to the original sliding surface. Then we get

$$
S_{c}=e+k_{0} \sigma
$$

where $S_{c}(e, \sigma)=0$ is the new sliding surface; $\sigma$ is conditional integrator with

$$
\dot{\sigma}=-k_{0} \sigma+\varepsilon \operatorname{sat}\left(\frac{S_{c}}{\varepsilon}\right), \quad \sigma(0) \leq \frac{\varepsilon}{k_{0}}
$$

$k_{0}>0$ is a tuning parameter and $\varepsilon>0$ is the width of the boundary layer.

Then we can get the new sliding control law as follows:

$$
\Delta M=\widehat{M}_{0}(\Omega)-I_{z} k \operatorname{sat}\left(\frac{S_{c}}{\varepsilon}\right) .
$$

This can ensure asymptotic regulation of yaw rate $\Omega$; the proof will be given as follows. To begin with, it is convenient to analyze the domain of $\sigma$ in Lemma 1 and the properties of $\operatorname{sat}\left(S_{c} / \varepsilon\right)$ in Lemma 2 and Theorem 3 below.

Lemma 1. Consider the set $D_{\sigma}=\left\{|\sigma| \leq \varepsilon / k_{0}\right\}$, under conditions (8); $D_{\sigma}$ is a positive invariant set.

Proof. The proof relies on the results in [17], replicated here for completeness. Using the Lyapunov function as $V=1 / 2 \sigma^{2}$, the time derivative is $\dot{V}=\sigma\left(-k_{0} \sigma+\varepsilon \operatorname{sat}\left(S_{c} / \varepsilon\right)\right)<-k_{0}|\sigma|(|\sigma|-$ $\left.\varepsilon / k_{0}\right)$. Then we know that, at the boundary of $D_{\sigma}, \dot{V}=0$. As $\sigma(0) \leq \varepsilon / k_{0}, D_{\sigma}$ is a positive invariant set.

Lemma 2. Outside the boundary layer, control law defined in (9) is equal to that defined in (6).

Proof. Outside the boundary layer, that is, $\left|S_{c}\right| \geq \varepsilon$, we get

$$
\operatorname{sat}\left(\frac{S_{c}}{\varepsilon}\right)=\operatorname{sgn}\left(S_{c}\right) \text {. }
$$

Therefore, Lemma 2 will be demonstrated if $\operatorname{sgn}\left(S_{c}\right)=$ $\operatorname{sgn}(e)$. From Lemma 1 , we know $k_{0}|\sigma| \leq \varepsilon$. Thus, we can get $\left|S_{c}\right|-k_{0}|\sigma| \geq 0$. If $S_{c}=e+k_{0} \sigma \geq 0$, then $e \geq k_{0}(|\sigma|-\sigma) \geq 0$; otherwise, if $S_{c}=e+k_{0} \sigma<0$, then $e<-k_{0}(|\sigma|+\sigma) \leq 0$. This equals $\operatorname{sgn}\left(S_{c}\right)=\operatorname{sgn}(e)$, so Lemma 2 is proved.

It can be seen from Lemma 2 that there is no difference between control law of SMC with and without conditional integrator before system entering the boundary layer, which assures the performance and robustness of SMC. 
When system enters the boundary layer, $\left|S_{c}\right|<\varepsilon$, we get

$$
\operatorname{sat}\left(\frac{S_{c}}{\varepsilon}\right)=\frac{S_{c}}{\varepsilon} \text {. }
$$

Then,

$$
\begin{gathered}
\dot{\sigma}=-k_{0} \sigma+\varepsilon \frac{S_{c}}{\varepsilon}=e, \\
\Delta M=\widehat{M}_{0}(\Omega)-\frac{k}{\varepsilon}\left(e+\int e d t\right) .
\end{gathered}
$$

Hence, the controller becomes a PI-like controller. The asymptotic conditions are established in Theorem 3 below.

Theorem 3. In the boundary layer, $(e, \sigma)$ converge to

$$
\left(\bar{e}=0, \bar{\sigma}=-\frac{\varepsilon}{k_{0} I_{z} k}\left[M_{0}\left(\Omega_{d}\right)-\widehat{M}_{0}\left(\Omega_{d}\right)\right]\right),
$$

when

$$
f(\Omega)=\frac{M_{0}(\Omega)-\widehat{M}_{0}(\Omega)}{I_{z}}
$$

Lipschitz on interval $\Omega \in\left(\Omega_{d}-\varepsilon, \Omega_{d}+\varepsilon\right)$.

Proof. From $\left|S_{c}\right|<\varepsilon$ we can get

$$
\begin{gathered}
\dot{e}=-\frac{1}{I_{z}}\left[k I_{z} \frac{e+k_{0} \sigma}{\varepsilon}+M_{0}\left(\Omega_{d}\right)-\widehat{M}_{0}\left(\Omega_{d}\right)\right], \\
\dot{\sigma}=e .
\end{gathered}
$$

Introducing $\widetilde{\sigma}$ and $\widetilde{S}_{c}$ as new variables where

$$
\begin{gathered}
\tilde{\sigma}=\sigma-\bar{\sigma}, \quad \widetilde{S}_{c}=S_{c}-\bar{S}_{c}, \\
\bar{S}_{c}=\bar{e}+k_{0} \bar{\sigma}=k_{0} \bar{\sigma} .
\end{gathered}
$$

Making use of the Lyapunov function

$$
V=\frac{1}{2} \widetilde{S}_{c}^{2}+\frac{1}{2} \widetilde{\sigma}^{2}
$$

The time derivative of $V$ is

$$
\begin{aligned}
\dot{V}= & \widetilde{S}_{c} \dot{\tilde{S}}_{c}+\widetilde{\sigma} \dot{\widetilde{\sigma}}=\widetilde{S}_{c} \dot{S}_{c}+\widetilde{\sigma} \dot{\sigma}=\widetilde{S}_{c}\left(\dot{e}+k_{0} \dot{\sigma}\right)+\widetilde{\sigma} e \\
= & \widetilde{S}_{c}\left\{-\frac{1}{I_{z}}\left[k I_{z} \frac{\widetilde{S}_{c}+\bar{S}_{c}}{\varepsilon}+M_{0}\left(\Omega_{d}\right)-\widehat{M}_{0}\left(\Omega_{d}\right)\right]\right\} \\
& +\widetilde{S}_{c} k_{0} e+\widetilde{\sigma} e \\
e= & \widetilde{S}_{c}-k_{0} \widetilde{\sigma}=S_{c}-k_{0} \bar{\sigma}-k_{0}(\sigma-\bar{\sigma})=S_{c}-k_{0} \sigma .
\end{aligned}
$$

Substituting (19) into (18) we get

$$
\begin{aligned}
\dot{V}= & -\left(\frac{k}{\varepsilon}-k_{0}\right) \widetilde{S}_{c}^{2}-\widetilde{S}_{c} \frac{1}{I_{z}}\left(k I_{z} \frac{\bar{S}_{c}}{\varepsilon}+M_{0}(\Omega)-\widehat{M}_{0}(\Omega)\right) \\
& -\left(k_{0}^{2}-1\right) \widetilde{S}_{c} \widetilde{\sigma}-k_{0} \widetilde{\sigma}^{2} .
\end{aligned}
$$

Makeup function is

$$
f(\Omega)=\frac{M_{0}(\Omega)-\widehat{M}_{0}(\Omega)}{I_{z}} .
$$

Under the condition that $f(\Omega)$ Lipschitz in the range of $\left(\Omega_{d}-\right.$ $\varepsilon, \Omega_{d}+\varepsilon$ ), we can get

$$
\left|f(\Omega)-f\left(\Omega_{d}\right)\right| \leq L|e|=L\left|\widetilde{S}_{c}-k_{0} \widetilde{\sigma}\right| \leq L\left|\widetilde{S}_{c}\right|+L k_{0}|\widetilde{\sigma}|,
$$

where $L$ is the Lipschitz constant. Then we get

$$
\begin{aligned}
\dot{V} \leq & -\left(\frac{k}{\varepsilon}-k_{0}\right) \widetilde{S}_{c}^{2}+k\left|\widetilde{S}_{c}\right|\left(L\left|\widetilde{S}_{c}\right|+L k_{0}|\widetilde{\sigma}|\right) \\
& -\left(k_{0}^{2}-1\right) \widetilde{S}_{c} \widetilde{\sigma}-k_{0} \widetilde{\sigma}^{2} \\
\leq & -\left(\frac{k}{\varepsilon}-k_{0}-k L\right) \widetilde{S}_{c}^{2}-k_{0} \widetilde{\sigma}^{2}+\left(k_{0}^{2}+1+L k k_{0}\right)\left|\widetilde{S}_{c}\right||\widetilde{\sigma}| \\
= & -\left[|\widetilde{\sigma}|\left|\widetilde{S}_{c}\right|\right]\left[\begin{array}{cc}
k_{0} & -\frac{k_{0}^{2}+1+L k k_{0}}{2} \\
-\frac{k_{0}^{2}+1+L k k_{0}}{2} & k\left(\frac{1}{\varepsilon}-L\right)-k_{0}
\end{array}\right] \\
& \times\left[\begin{array}{c}
|\widetilde{\sigma}| \\
\left.\left|\widetilde{S}_{c}\right|\right]
\end{array}\right.
\end{aligned}
$$

Therefore, left side of (23) will be negative definite when the conditions below are met:

(A) $k_{0}>0$;

(B)

$$
k_{0}\left[k\left(\frac{1}{\varepsilon}-L\right)-k_{0}\right]-\left[\frac{\left(k_{0}^{2}+1+L k k_{0}\right)}{2}\right]^{2}>0 .
$$

Condition (A) will be ensured by selecting a positive $k_{0}$, while condition (B) will be verified when

$$
\varepsilon \leq \frac{1}{L+\left(k_{0} / k\right)+\left(1 / k k_{0}\right)\left[\left(k_{0}^{2}+1+L\right) / 2\right]^{2}} .
$$

This can be ensured by choosing an appropriate $\varepsilon$. Hence, with properly selecting $k_{0}$ and $\varepsilon$, the system state will asymptotically converge to $\left(\widetilde{\sigma}=0, \widetilde{S}_{c}=0\right)$ which is equivalent to (13).

One thing to note during the proof is the requirement of $f(\Omega)$ Lipschitz on $\left(\Omega_{d}-\varepsilon, \Omega_{d}+\varepsilon\right)$. As $M_{0}(\omega)$ is continuous and bounded, $f(\Omega)$ can be sufficiently smooth; therefore the requirement is fulfilled.

In light of the discussions above, the SMC\&CI based controller has been built. The desired yaw moment can be calculated by (9), in which $k, k_{0}$, and $\varepsilon$ are tuning parameters. $k$ defines the convergence speed of the control system and should be given a relatively big value for giving some extra 
margin for the modeling error. $k_{0}$ affects the convergence speed when system enters the boundary layer; a bigger $k_{0}$ will enlarge the influence of the conditional integrator; it should be increased gradually due to the controller's performance. As the system's asymptotic stability is obtained when $\varepsilon$ goes to zero, $\varepsilon$ should be gradually reduced until the transient performance is satisfactory, as suggested in [17].

\section{Lower-Level Controller Design}

In this section, preliminary results regarding the design of the lower-level controller will be presented. The desired yaw moment will be allocated to tires' slip ratio targets.

Regulating contact forces between road and tires is usually used as means to control vehicle motion. Differential driving/braking on the FWIA electric ground vehicles can change the yaw moment from road surface around the vehicle $z$-axis. Yaw stability control system needs to decide the wheel driving/braking torque according to the desired yaw moment $\Delta M$. Slip ratio can connect wheel torque with tire-road forces. As accurate regulation of tire longitudinal and lateral forces can be realized by slip ratio adjustment, instead of calculating desired tire forces from desired yaw moment, we calculate the desired wheel slip ratio directly due to the mathematical relationship between wheels' slip ratio and yaw moment. In the second layer of the proposed algorithm, wheels' slip ratios are determined.

3.1. Relationship between Yaw Moment and Wheel Slip Ratio. Take the left wheel on front axle shown in Figure 2 as an example; the relationship between tire forces $\left(F_{\mathrm{FLx}}\right.$ stands for longitudinal force and $F_{\mathrm{FL} y}$ is lateral force) and yaw moment around $z$-axis at vehicle gravity center is written as follows:

$$
\begin{aligned}
M_{\mathrm{FL}}= & F_{\mathrm{FL} x}\left(a \sin \delta_{\mathrm{FL}}-\frac{d_{\mathrm{F}}}{2} \cos \delta_{\mathrm{FL}}\right) \\
& +F_{\mathrm{FL} y}\left(\frac{d_{\mathrm{F}}}{2} \sin \delta_{\mathrm{FL}}+a \cos \delta_{\mathrm{FL}}\right),
\end{aligned}
$$

where $M_{\mathrm{FL}}$ is the yaw moment around $z$-axis at vehicle gravity center caused by forces from the tire-road contact surface of front left wheel and $a, d_{\mathrm{F}}$, and $\delta_{\mathrm{FL}}$ are the distance from vehicle gravity center to the front axle, front track, and front left wheel steering angle, respectively.

According to the magic formula tire model in [18], we get

$$
\begin{aligned}
& F_{\mathrm{FL} x}=f_{\mathrm{FL} x}\left(F_{\mathrm{FL} z}, \alpha_{\mathrm{FL}}, \lambda_{\mathrm{FL}}\right), \\
& F_{\mathrm{FL} y}=f_{\mathrm{FL} y}\left(F_{\mathrm{FL} z}, \alpha_{\mathrm{FL}}, \lambda_{\mathrm{FL}}\right) .
\end{aligned}
$$

Substituting (27) and (28) into (26) and calculating the $\lambda_{\mathrm{FL}}$ partial differential we get

$$
\begin{aligned}
\Delta M_{\mathrm{FL}}= & \frac{\partial F_{\mathrm{FL} x}}{\partial \lambda_{\mathrm{FL} 0}}\left(a \sin \delta_{\mathrm{FL}}-\frac{d_{\mathrm{F}}}{2} \cos \delta_{\mathrm{FL}}\right) \\
& \left.+\frac{\partial F_{\mathrm{FL} y}}{\partial \lambda_{\mathrm{FL} 0}}\left(\frac{d_{\mathrm{F}}}{2} \sin \delta_{\mathrm{FL}}+a \cos \delta_{\mathrm{FL}}\right)\right] \Delta \lambda_{\mathrm{FL}} .
\end{aligned}
$$

Equation (27) gives the quantitative relation between wheel slip ratio and yaw moment imposed to vehicle at the operating point. Similarly,

$$
\begin{aligned}
\Delta M_{\mathrm{FR}}= & \frac{\partial F_{\mathrm{FR} x}}{\partial \lambda_{\mathrm{FR} 0}}\left(a \sin \delta_{\mathrm{FR}}+\frac{d_{\mathrm{F}}}{2} \cos \delta_{\mathrm{FR}}\right) \\
& \left.+\frac{\partial F_{\mathrm{FR} y}}{\partial \lambda_{\mathrm{FR} 0}}\left(-\frac{d_{\mathrm{F}}}{2} \sin \delta_{\mathrm{FR}}+a \cos \delta_{\mathrm{FR}}\right)\right] \Delta \lambda_{\mathrm{FR}} \\
\Delta M_{\mathrm{RL}}= & {\left[\frac{\partial F_{\mathrm{RL} x}}{\partial \lambda_{\mathrm{RL} 0}}\left(-\frac{d_{\mathrm{R}}}{2}\right)+\frac{\partial F_{\mathrm{RL} y}}{\partial \lambda_{\mathrm{RL} 0}}(-b)\right] \Delta \lambda_{\mathrm{RL}} } \\
\Delta M_{\mathrm{RR}}= & {\left[\frac{\partial F_{\mathrm{RR} x}}{\partial \lambda_{\mathrm{RR} 0}}\left(\frac{d_{\mathrm{R}}}{2}\right)+\frac{\partial F_{\mathrm{RR} y}}{\partial \lambda_{\mathrm{RR} 0}}(-b)\right] \Delta \lambda_{\mathrm{RR}} }
\end{aligned}
$$

where corner marks FR, RL, and RR stand for the front right, rear left, and rear right wheel, respectively; $b$ and $d_{\mathrm{R}}$ are the distance from vehicle gravity center to the rear axle and rear track, respectively.

We can get the relationship between $\Delta M$ and $\Delta \lambda$ from (27), (28), (29), and (30):

$$
\Delta M=\sum_{i=1}^{4} \frac{\partial M_{i}}{\partial \lambda_{i 0}} \Delta \lambda_{i}
$$

where $i=1, \ldots, 4$ represent the FL, FR, RL, and RR wheel, respectively. At the operating point, a kind of proportionate relationship between desired yaw moment and wheel slip ratio is established that can be used in calculating slip ratio according to the desired yaw moment.

3.2. Control Boundaries of Wheel Slip Ratio. It is important to mention that $\partial M / \partial \lambda_{0}$ calculated by (33) is only effective around the present tire state $\left(F_{z i 0}, \lambda_{i 0}, \alpha_{i 0}\right)$, where $F_{z i 0}$ is the vertical tire force and $\alpha_{i 0}$ is the tire side slip angle at the operating point. This is due to the nonlinear relationship between tire longitudinal force $F_{x}$ /lateral force $F_{y}$ and slip ratio $\lambda$. The control boundaries of slip ratio which are critical for avoiding wrong interference of braking/driving torque should be established; the process is shown as follows.

Without loss of generality, we take the left turning vehicle shown in Figure 2 as an example to show the process. If the desired yaw moment is positive, that is, $\Delta M>0$, braking torque will be applied onto the RL wheel preferentially provided that $\partial M_{R L} / \partial \lambda_{R L 0}$ is negative. We can get the following from (29):

$$
\frac{\partial M_{\mathrm{RL} x}}{\partial \lambda_{\mathrm{RL} 0}}=\left[\frac{\partial F_{\mathrm{RL} x}}{\partial \lambda_{\mathrm{RL} 0}}\left(-\frac{d_{\mathrm{R}}}{2}\right)+\frac{\partial F_{\mathrm{RL} y}}{\partial \lambda_{\mathrm{RL} 0}}(-b)\right] .
$$

Figure 3 shows how $\partial M_{\mathrm{RL}} / \partial \lambda_{\mathrm{RL} 0}$ changes with slip ratio $\lambda_{\mathrm{RL}}$, when the RL tire is in the following state: $F_{z \mathrm{RL}}=4100 \mathrm{~N}$, $\alpha_{\mathrm{RL}}=-5 \mathrm{deg}$. From Figure 3 we can see that $M_{\mathrm{RL}}$ will always increase when slip ratio decreases. So the lower bound for the $\mathrm{RL}$ wheel slip ratio should be calculated by

$$
\frac{\partial F_{\mathrm{RL} x}}{\partial \lambda_{\mathrm{RL}}}=0
$$




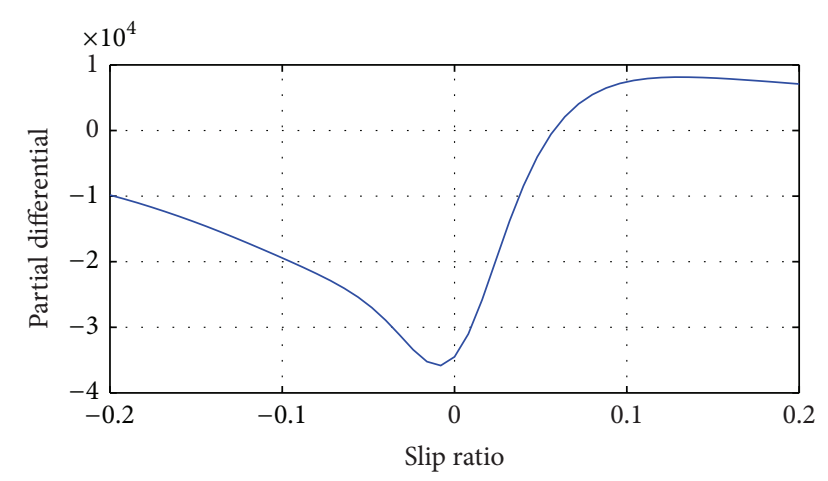

FIgURe 3: Partial derivative of the yaw moment versus the RL wheel slip.

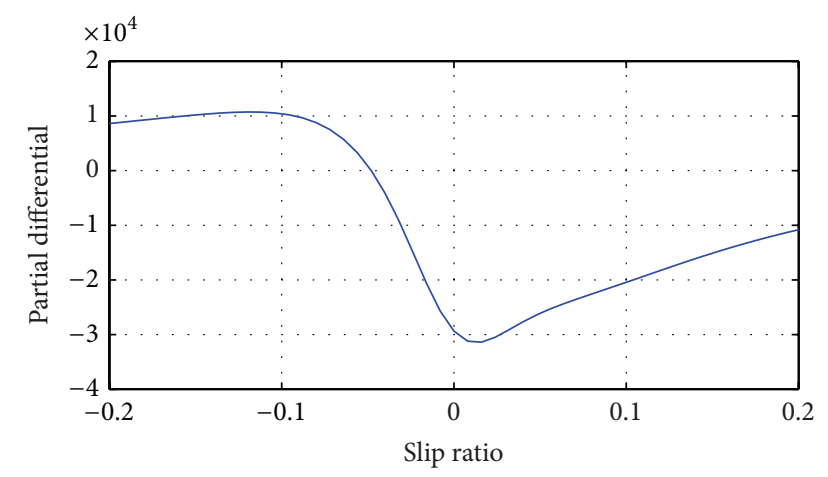

FIGURE 4: Partial derivative of the yaw moment versus the FL wheel slip.

where $F_{\mathrm{RL} x}$ is the braking force from the tire-road contact surface. This ensures the tire staying in the linear area. As braking torque will be applied to the RL wheel, there is no need to calculate the upper bound of the slip ratio.

If application of braking torque on the RL wheel is not enough to generate the desired yaw moment, driving torque will be imposed to the RR wheel. Therefore, we need to calculate the upper bound of slip ratio of the RR wheel. In the similar way, the upper bound should be calculated by

$$
\frac{\partial F_{\mathrm{RR} x}}{\partial \lambda_{\mathrm{RR}}}=0,
$$

where $F_{\mathrm{RR} x}$ is the driving force from road surface.

Things will be different when calculating boundaries of slip ratios of FL and FR tires, when differential braking/driving on the rear axle cannot generate the desired yaw moment. Take the braking of FL wheel as an example; longitudinal braking force from the road surface will increase when slip ratio gets smaller, which generates a bigger yaw moment $M_{\mathrm{FL}}$. At the same time, lateral force will decrease which reduces $M_{\mathrm{FL}}$. That is to say, as shown in Figure $4, M_{\mathrm{FL}}$ is not monotonic increasing when slip ratio is decreasing. With this in mind, the lower bound of FL wheel slip ratio is calculated by

$$
\frac{\partial M_{\mathrm{FL}}}{\partial \lambda_{\mathrm{FL}}}=0, \quad \frac{\partial F_{\mathrm{FL} x}}{\partial \lambda_{\mathrm{FL}}} \frac{\partial F_{\mathrm{FL} y}}{\partial \lambda_{\mathrm{FL}}}<0 .
$$

TABLE 1: Vehicle system parameters.

\begin{tabular}{lcc}
\hline Vehicle mass & $M$ & $1486 \mathrm{~kg}$ \\
Yaw moment of inertia & $I_{z}$ & $2023 \mathrm{~kg} / \mathrm{m}^{2}$ \\
c.g. distance to front axle & $a$ & $1.016 \mathrm{~m}$ \\
c.g. distance to rear axle & $b$ & $1.562 \mathrm{~m}$ \\
Front track width & $d_{\mathrm{F}}$ & $1.539 \mathrm{~m}$ \\
Rear track width & $d_{\mathrm{R}}$ & $1.544 \mathrm{~m}$ \\
Wheel radius & $r$ & $0.298 \mathrm{~m}$ \\
\hline
\end{tabular}

The upper bound of FR wheel slip ratio can be calculated in similar way:

$$
\frac{\partial M_{\mathrm{FR}}}{\partial \lambda_{\mathrm{FR}}}=0, \quad \frac{\partial F_{\mathrm{FR} x}}{\partial \lambda_{\mathrm{FR}}} \frac{\partial F_{\mathrm{FR} y}}{\partial \lambda_{\mathrm{FR}}}<0 .
$$

In light of the analysis above, we can get the upper or lower bound of slip ratio, which will be used as constraint for slip ratio. In the same way, we can get boundaries for slip ratios under other conditions.

At this point, the hierarchical yaw stability control algorithm has been developed. Although the higher-layer controller has been designed with nonlinear techniques, it can be implemented as a simple PI controller with antiwindup. The desired yaw moment is allocated to wheel slip ratio directly, which is convenient for grasping control boundaries of actuators.

\section{Simulation Results and Discussions}

The algorithm developed in this paper was evaluated in a cosimulation between a vehicle dynamics simulator, the Carsim, and Matlab/Simulink.

4.1. Simulation Platform Introduction. The commercial vehicle dynamics simulation package, Carsim, was used to provide a C-class hatchback car test platform. Models of the electric powertrains and the control strategies were implemented in Matlab/Simulink. Figure 5 shows the structure of the cosimulation platform. The vehicle system parameters are shown in Table 1.

4.2. The Electric Powertrain Model. In this paper, the powertrain was modelled with bench tests. According to analysis in [19], an electric motor and its drive can be simply modelled as follows:

$$
G(s)=\frac{T_{m}}{T_{m}^{*}}=\frac{1}{1+2 \xi s+2 \xi^{2} s^{2}},
$$

where $T_{m}$ is the driving torque output by the powertrain, $T_{m}^{*}$ is the torque command received by the motor controller, and $\xi$ is a constant mainly determined by motor's characteristics and will be obtained by experiments.

Take the time delay caused by information transmission and gear clearance into consideration; a delay element was added to (39); then we get a revised model as follows:

$$
G^{\prime}(s)=\frac{T_{m}}{T_{m}^{*}}=\frac{1}{1+2 \xi s+2 \xi^{2} s^{2}} e^{-\tau s} .
$$




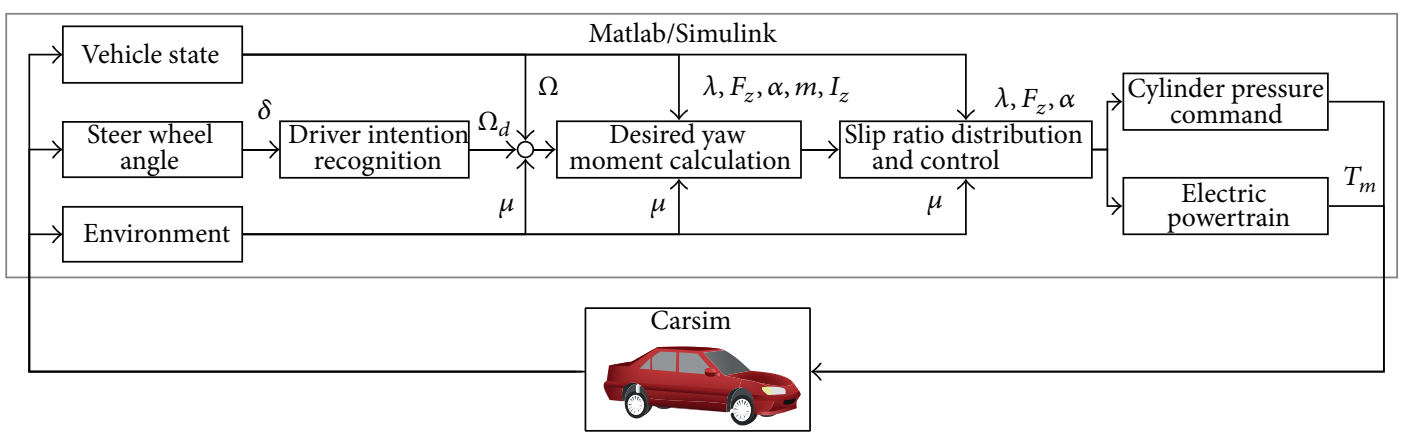

Figure 5: Schematic diagram of the Carsim-Matlab cosimulation.

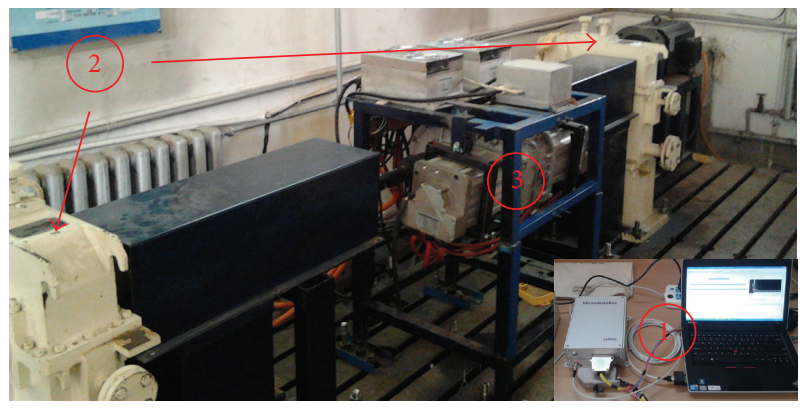

FIGURE 6: Test bench built for modelling the powertrain.

TABLE 2: Electric powertrain parameters.

\begin{tabular}{lc}
\hline Rated power & $8 \mathrm{kw}$ \\
Rated speed & $2960 \mathrm{rpm}$ \\
Rated torque & $26 \mathrm{Nm}$ \\
Peak torque & $78 \mathrm{Nm}$ \\
Maximum speed & $8000 \mathrm{rpm}$ \\
Gearing efficiency & $96 \%$ \\
Ratio of gear reducer & 0.7 \\
\hline
\end{tabular}

A bench shown in Figure 6 had been built and used to get powertrain outputs from different torque commands. In Figure 6, (1) is the monitoring system, (2) is the dynamometer system with speed sensor and torque sensor, and (3) is a dual-motor independently driving system used for driving the front or rear axle and its parameters are shown in Table 2.

Figure 7 is an example of model outputs and measured outputs compared with torque commands.

4.3. Simulation Results and Discussions. The yaw stability control approach proposed in this paper consists of two layers: the desired yaw moment calculation layer and the slip ratio distribution and control layer. Two different scenarios have been simulated for the verification of the above two layers, respectively.

Scenario 1 was mainly used to verify the SMC\&CI based controller in the higher layer of the algorithm. The SMC and SMC with saturation function, or say continuous sliding mode control (CSMC) based controllers, were used as comparisons. When the vehicle was running on the low

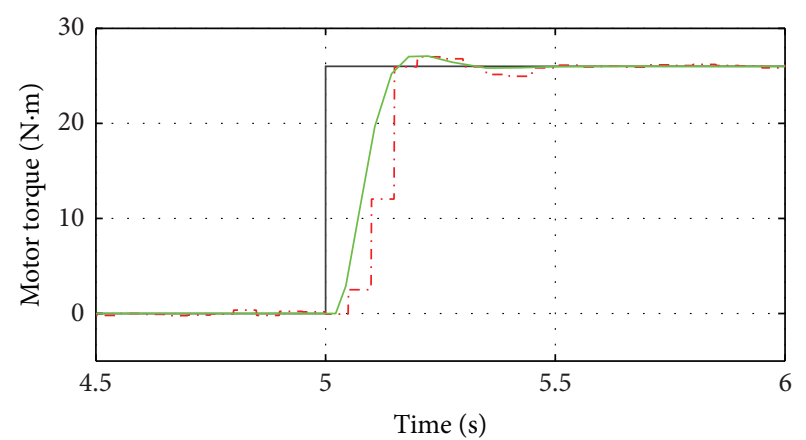

_ Torque command
_- Measured value
_ Model output

Figure 7: Model output versus measured output.

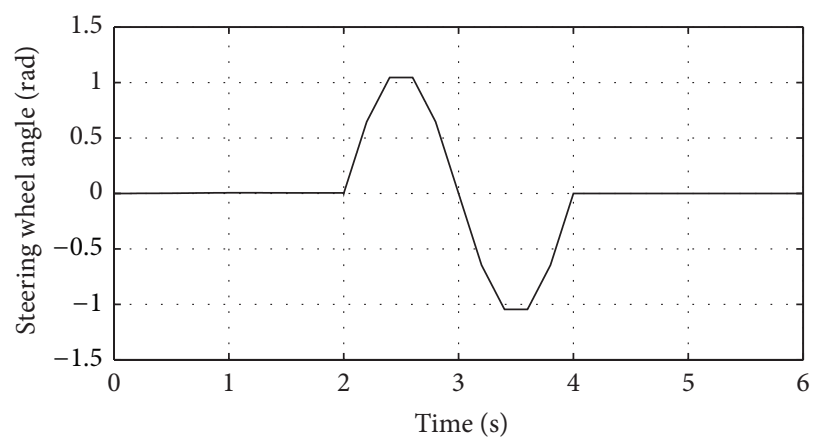

FIGURE 8: Steering wheel input.

friction road (the adhesion coefficient is 0.4 ), a lane change maneuver was performed. There was no accelerator or brake pedal input during the simulations. The steering wheel angle is shown in Figure 8, and vehicle initial velocity is $90 \mathrm{~km} / \mathrm{h}$.

Simulation results are shown from Figures 9-13. The desired yaw moment generated by the SMC based controller during the lane change maneuver is shown in Figure 9; there exist obvious chattering phenomena. After the saturation function or conditional integrator was added to the controller, the chattering issue was eliminated as shown in Figure 10. Moreover, it can be seen from Figure 11 that the SMC\&CI based controller can enhance the vehicle tracking 


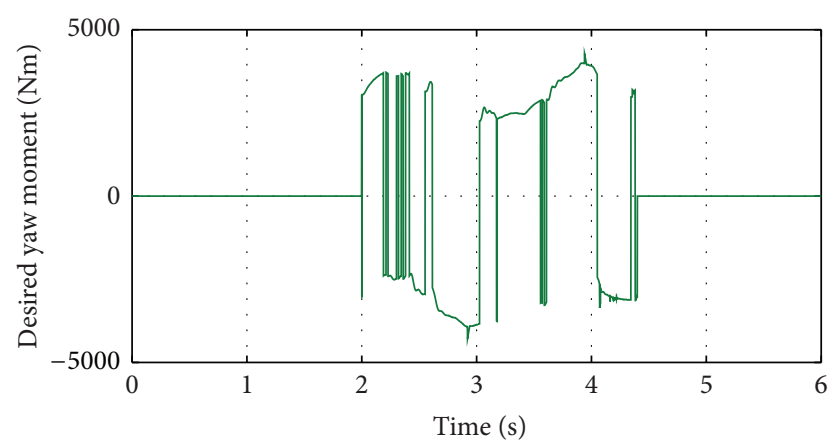

FIGURE 9: Desired yaw moment (SMC based controller).

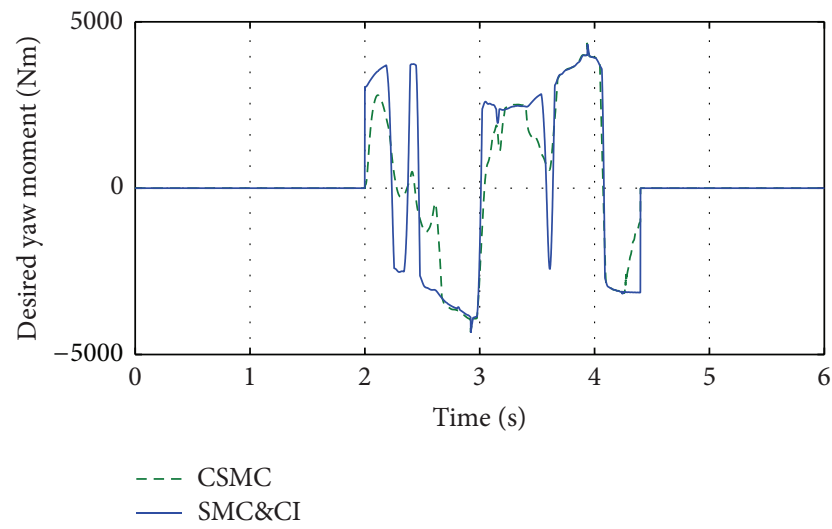

Figure 10: Desired yaw moment (CSMC/SMC\&CI based controller).

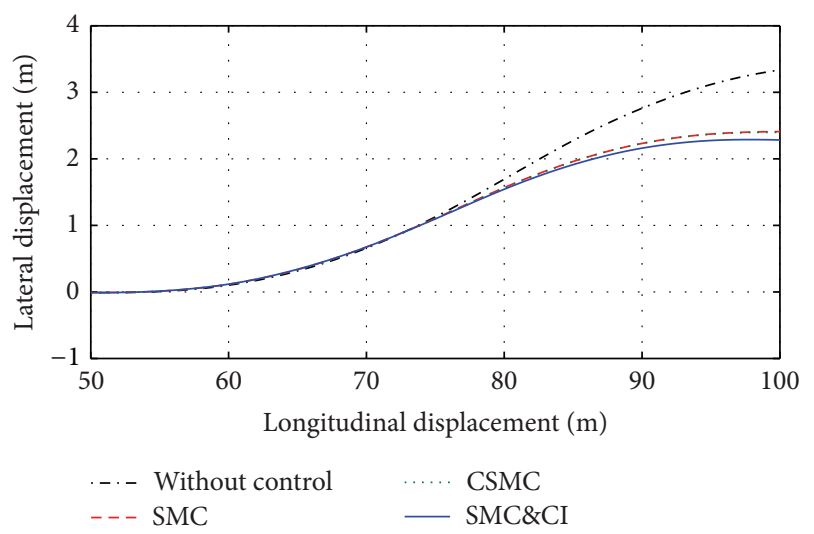

FIGURE 11: Trajectory of the vehicle c.g.

ability compared to the SMC and CSMC based ones. This is a result of the smaller yaw rate tracking error when SMC\&CI is adopted, as shown in Figure 12. Moreover, the SMC\&CI based controller has the same influence as CSMC based one on the vehicle longitudinal velocity, as shown in Figure 13.

Scenario 2 was implemented to verify the whole control algorithm presented in this paper. And the ESC program contained in the Carsim simulation package was used as comparison. The ISO3888-1:1999 double lane change maneuver was performed, and there was no accelerator or brake pedal

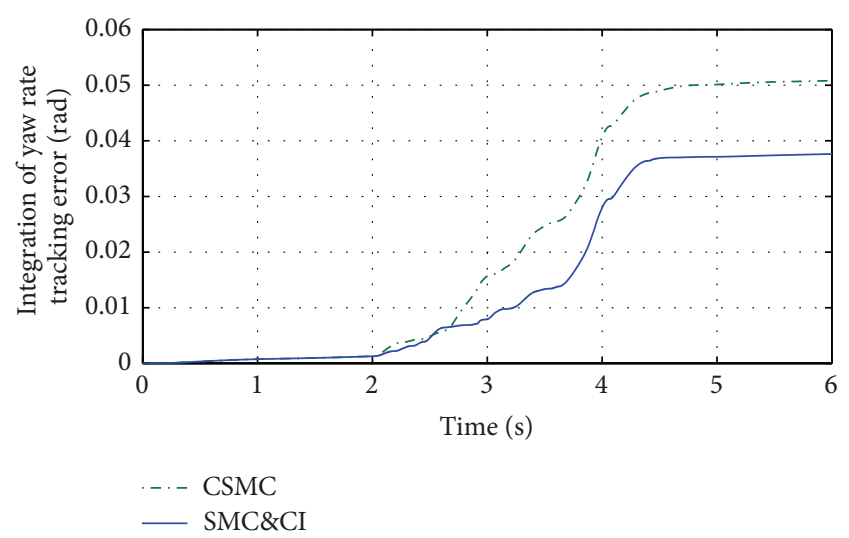

FIGURE 12: Integration of yaw rate tracking error.

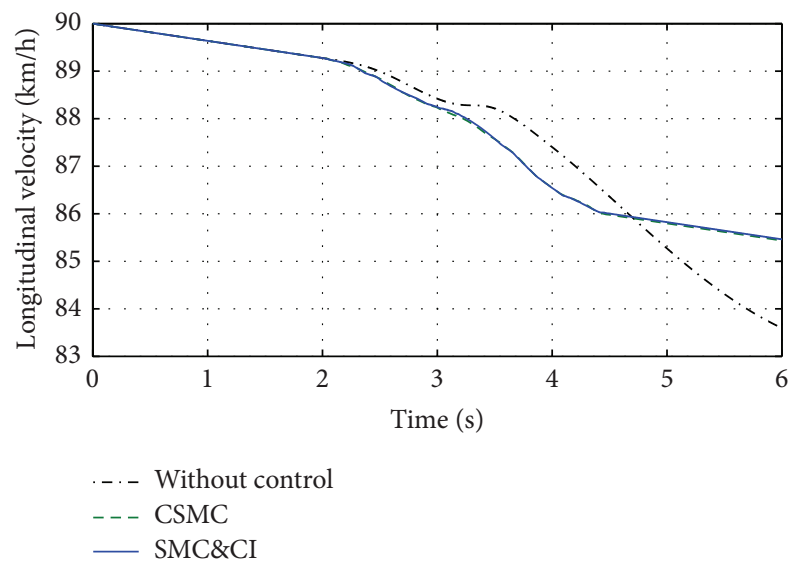

FIGURE 13: Longitudinal velocity of the vehicle c.g.

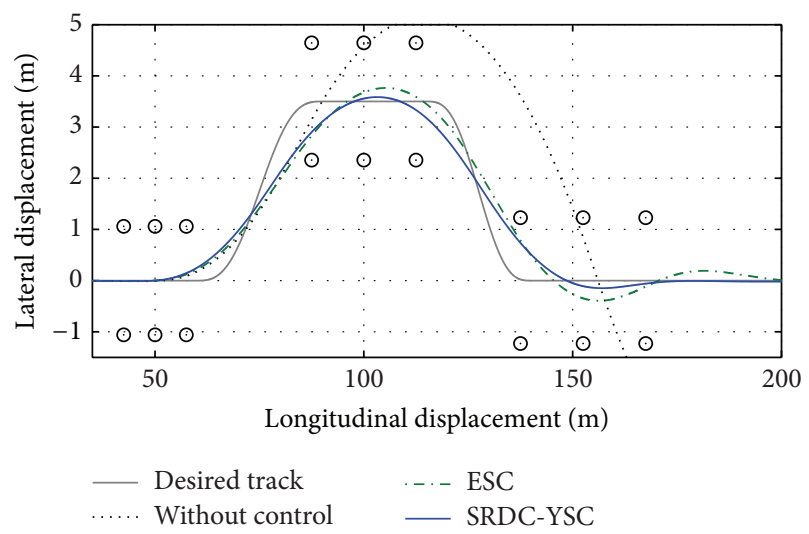

FIGURE 14: Trajectory of the vehicle c.g.

input during the process. Vehicle initial velocity is $90 \mathrm{~km} / \mathrm{h}$, and road adhesion coefficient is 0.4 .

Simulation results are shown from Figures 14-21. In those figures, SRDC-YSC (slip ratio distribution and control-based yaw stability control) represents the proposed algorithm, and ESC represents the yaw motion control program in Carsim.

It can be seen from Figures 14 and 15 that the vehicle tracking ability has been enhanced by SRDC-YSC with 


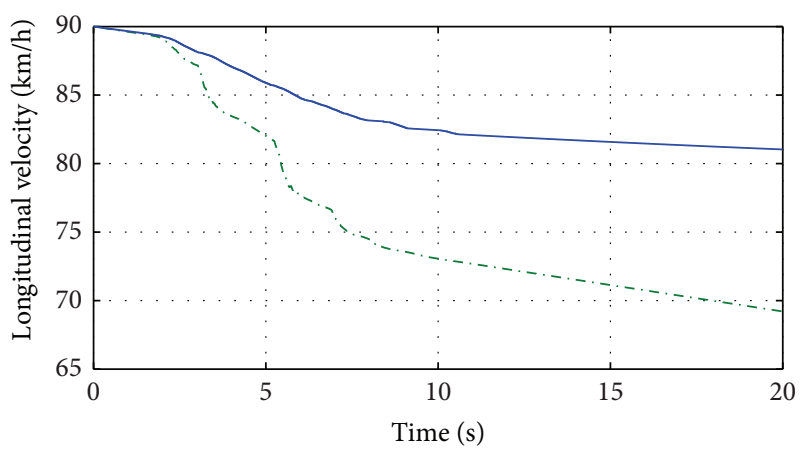

-. - ESC

- SRDC-YSC

FIGURE 15: Longitudinal velocity of the vehicle c.g.

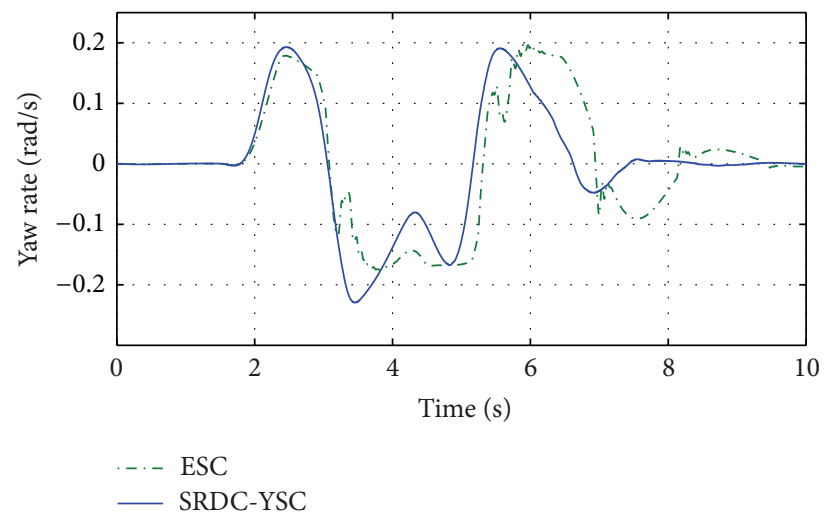

FIGURE 16: Vehicle yaw rate.

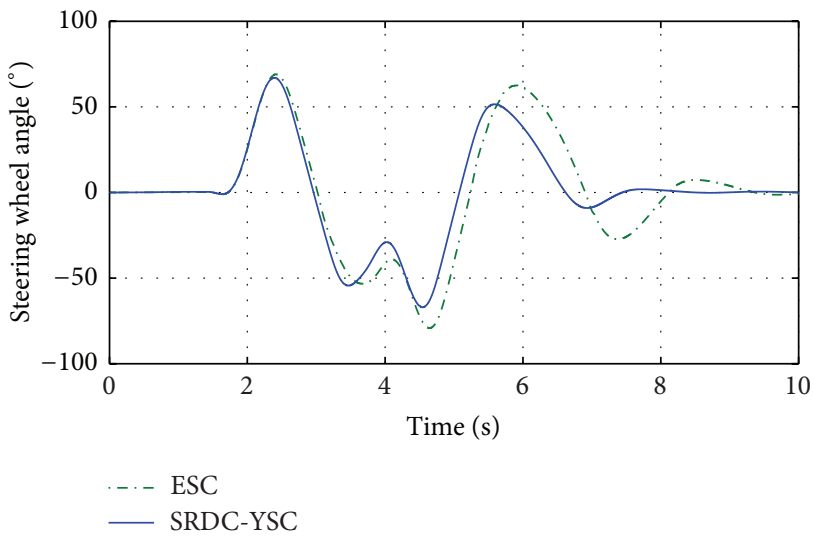

FIGURE 17: Steering wheel input.

a smaller velocity decline than ESC. Figure 16 shows the history of the vehicle yaw rate; the one generated by SRDCYSC is much smoother which ensures a better driving feeling. And we can see from Figure 17 that SRDC-YSC alleviates the driver's workload. Tire slip angle that represents the level of vehicle lateral stability shown in Figures 18 and 19 has verified the effectiveness of the proposed algorithm. This is because the yaw moment applied to the vehicle from

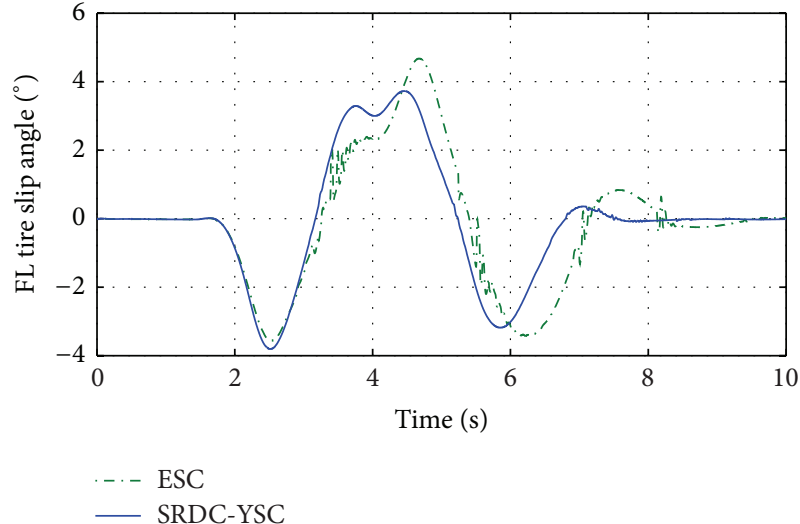

FIGURE 18: FL tire slip angle.

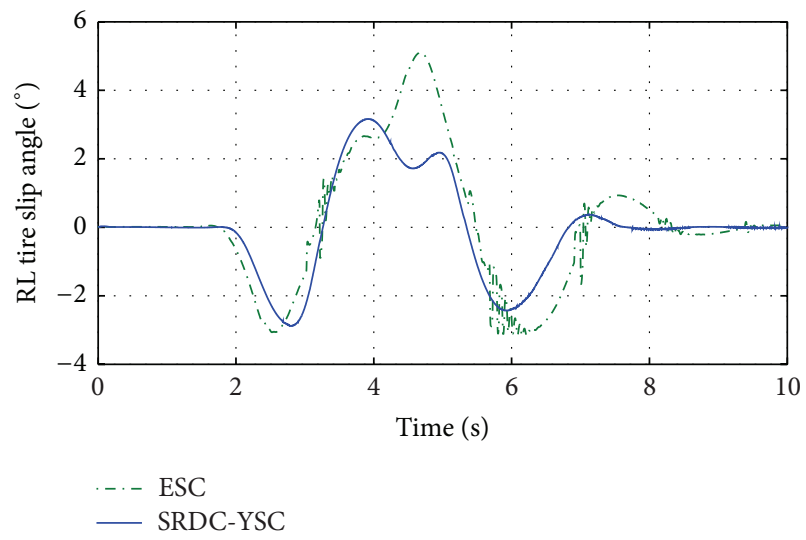

FIGURE 19: RL tire slip angle.

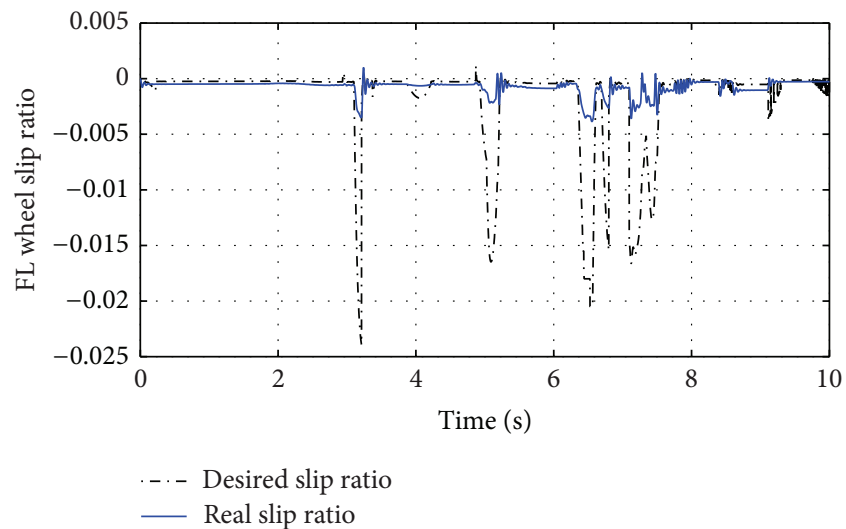

FIGURE 20: FL wheel slip ratio.

the road surface with SRDC-YSC is better manipulated than with ESC. As the higher layer of those two algorithms is the same, allocating the control effort to wheel slip ratio according to a dynamic relation between yaw moment and slip ratio makes the yaw moment more accurately complied. The history of slip ratio versus command has been shown in Figures 20 and 21; conclusions can be drawn that the RL wheel slip ratio changes more obvious than that of the FL wheel 


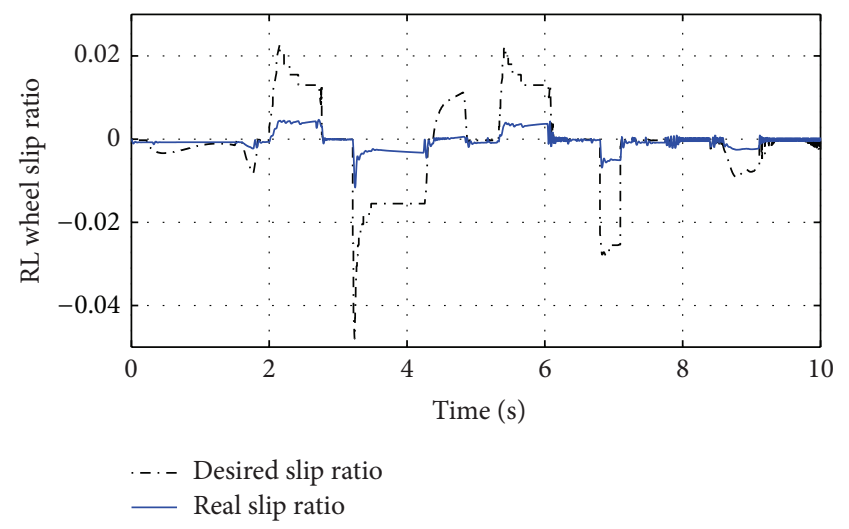

FIGURE 21: RL wheel slip ratio.

which is a result of the lower side slip angle of the rear axle and the desired yaw moment is allocated to this side preferentially.

\section{Conclusions}

A hierarchical yaw stability control algorithm for FWIA electric ground vehicles is presented. A sliding mode approach based on conditional integrator was proposed to control the vehicle yaw rate. It keeps the characteristics of sliding mode control and when system enters the boundary layer, it turns to be a PI-like controller. The asymptotic stability property was demonstrated through the Lyapunov method. In the lower layer of the control system, the required yaw moment is allocated to the wheel slip ratio instead of tire forces. The magic formula tire model was used to establish a dynamic relation between yaw moment and wheel slip ratio. Simulations under various driving scenarios were carried out with a high-fidelity, Carsim, and full vehicle model. Results verified the effectiveness of the proposed approach.

\section{Conflict of Interests}

The authors declare that there is no conflict of interests regarding the publication of this paper.

\section{Acknowledgment}

The authors acknowledge that this paper has been supported by National Natural Science Foundation of China (no. 51175043).

\section{References}

[1] S. Horiuchi, "Evaluation of chassis control method through optimisation-based controllability region computation," Vehicle System Dynamics, vol. 50, no. 1, pp. 19-31, 2012.

[2] J. Kim, C. Park, S. Hwang, Y. Hori, and H. Kim, "Control algorithm for an independent motor-drive vehicle," IEEE Transactions on Vehicular Technology, vol. 59, no. 7, pp. 3213-3222, 2010.

[3] L. Feiqiang, W. Jun, and L. Zhaodu, "Motor torque based vehicle stability control for four-wheel-drive electric vehicle," in
Proceedings of the IEEE Vehicle Power and Propulsion Conference (VPPC '09), pp. 1596-1601, Dearborn, Mich, USA, September 2009.

[4] P. He and Y. Hori, "Optimum traction force distribution for stability improvement of 4WD EV in critical driving condition," in Proceedings of the 9th IEEE International Workshop on Advanced Motion Control, pp. 596-601, Istanbul, Turkey, March 2006.

[5] Z. He and X. Ji, "Nonlinear robust control of integrated vehicle dynamics," Vehicle System Dynamics, vol. 50, no. 2, pp. 247-280, 2012.

[6] P. Falcone, H. Eric Tseng, F. Borrelli, J. Asgari, and D. Hrovat, "MPC-based yaw and lateral stabilisation via active front steering and braking," Vehicle System Dynamics, vol. 46, no. 1, pp. 611-628, 2008.

[7] R. Wang, H. R. Karimi, N. Chen, G. Yin, and J. Wang, "Motion control of four-wheel independently actuated electric ground vehicles considering Tire force saturations," Mathematical Problems in Engineering, vol. 2013, Article ID 819302, 8 pages, 2013.

[8] J. Ahmadi, A. K. Sedigh, and M. Kabganian, "Adaptive vehicle lateral-plane motion control using optimal tire friction forces with saturation limits consideration," IEEE Transactions on Vehicular Technology, vol. 58, no. 8, pp. 4098-4107, 2009.

[9] J. Wang and R. G. Longoria, "Coordinated and reconfigurable vehicle dynamics control," IEEE Transactions on Control Systems Technology, vol. 17, no. 3, pp. 723-732, 2009.

[10] Y. Chen, J. K. Hedrick, and K. Guo, "A novel direct yaw moment controller for in-wheel motor electric vehicles," Vehicle System Dynamics, vol. 51, no. 6, pp. 925-942, 2013.

[11] R. P. Osborn and T. Shim, "Independent control of all-wheeldrive torque distribution," Vehicle System Dynamics, vol. 44, no. 7, pp. 529-546, 2006.

[12] F. Tahami, S. Farhangi, and R. Kazemi, "A fuzzy logic direct yawmoment control system for all-wheel-drive electric vehicles," Vehicle System Dynamics, vol. 41, no. 3, pp. 203-221, 2004.

[13] S. Horiuchi, K. Okada, and S. Nohtomi, "Improvement of vehicle handling by nonlinear integrated control of four wheel steering and four wheel torque," JSAE review, vol. 20, no. 4, pp. 459-464, 1999.

[14] J. Limroth, Real-Time Vehicle Parameter Estimation, Clemson University, Clemson, SC, USA, 2009.

[15] R. De Castro, R. E. Araujo, and D. Freitas, "Wheel slip control of EVs based on sliding mode technique with conditional integrators," IEEE Transactions on Industrial Electronics, vol. 60, no. 8, pp. 3256-3271, 2013.

[16] H. Khalil, Nonlinear Systems, Prentice-Hall, Englewood Cliffs, NJ, USA, 3rd edition, 2002.

[17] S. Seshagiri and H. K. Khalil, "Robust output feedback regulation of minimum-phase nonlinear systems using conditional integrators," Automatica, vol. 41, no. 1, pp. 43-54, 2005.

[18] H. B. Pacejka, Tyre and Vehicle Dynamics, Oxford, UK, Butterworth Heinemann, 2002.

[19] F. Tahami, R. Kazemi, and S. Farhanghi, "A novel driver assist stability system for all-wheel-drive electric vehicles," IEEE Transactions on Vehicular Technology, vol. 52, no. 3, pp. 683-692, 2003. 


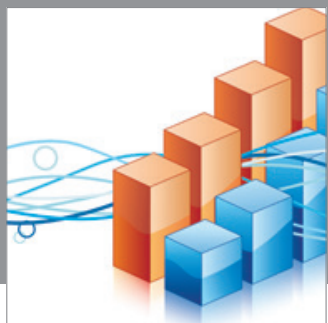

Advances in

Operations Research

mansans

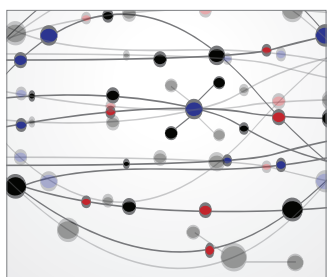

The Scientific World Journal
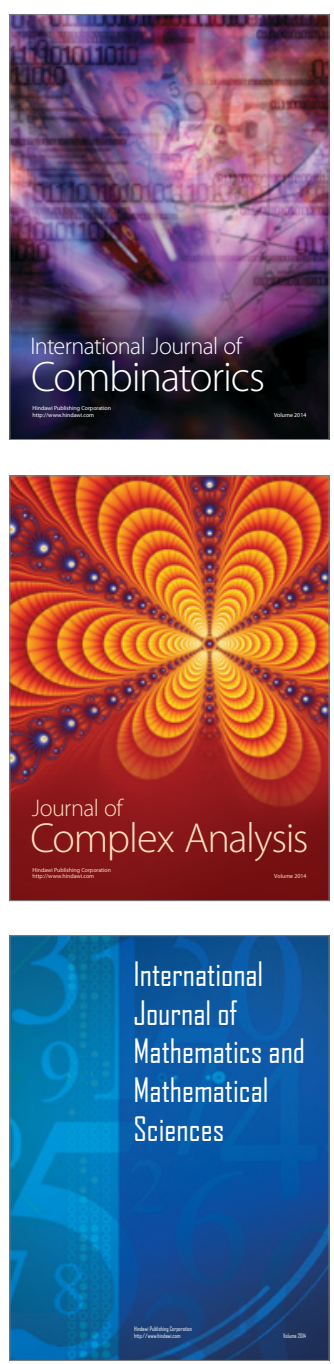
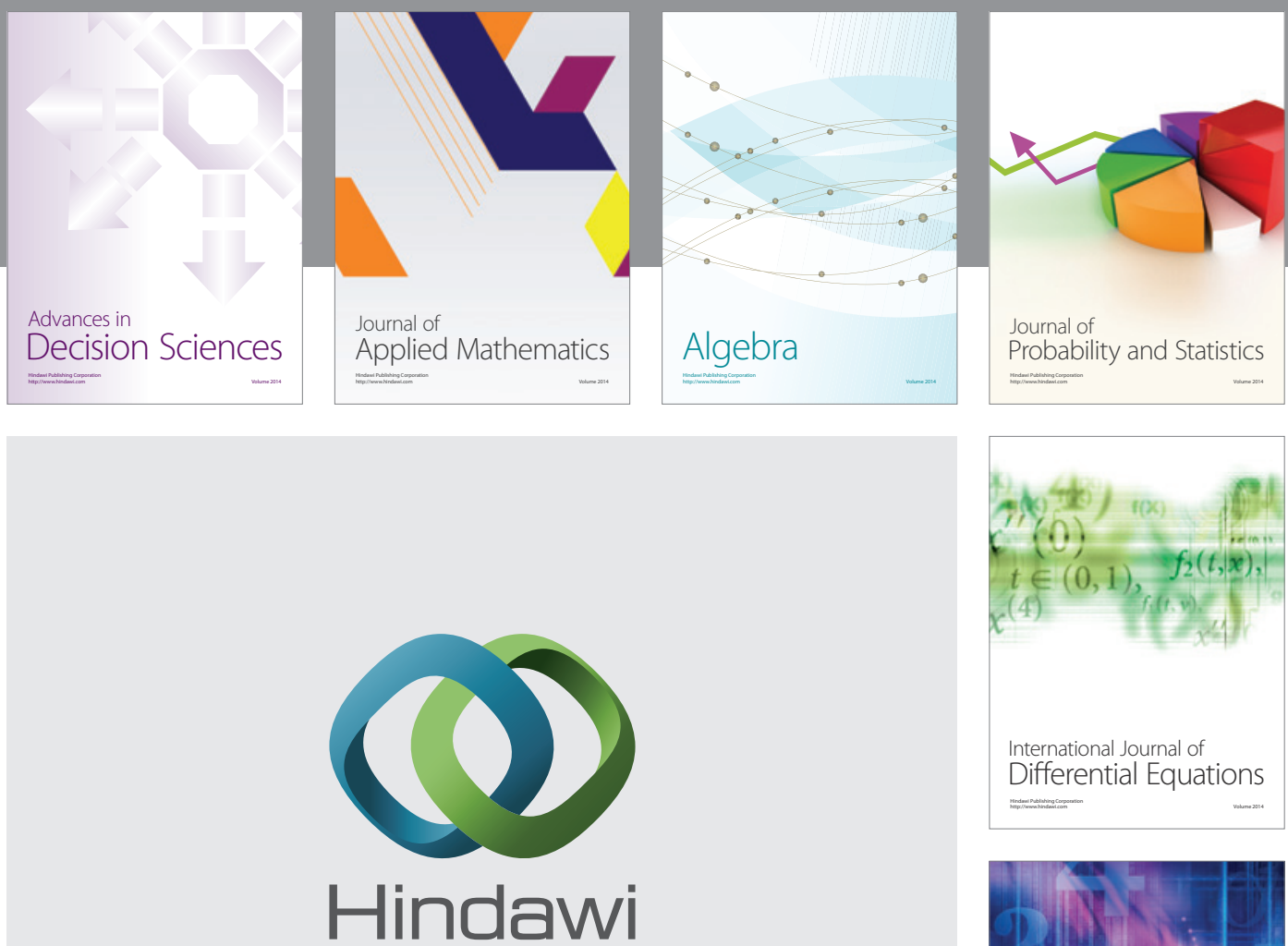

Submit your manuscripts at http://www.hindawi.com
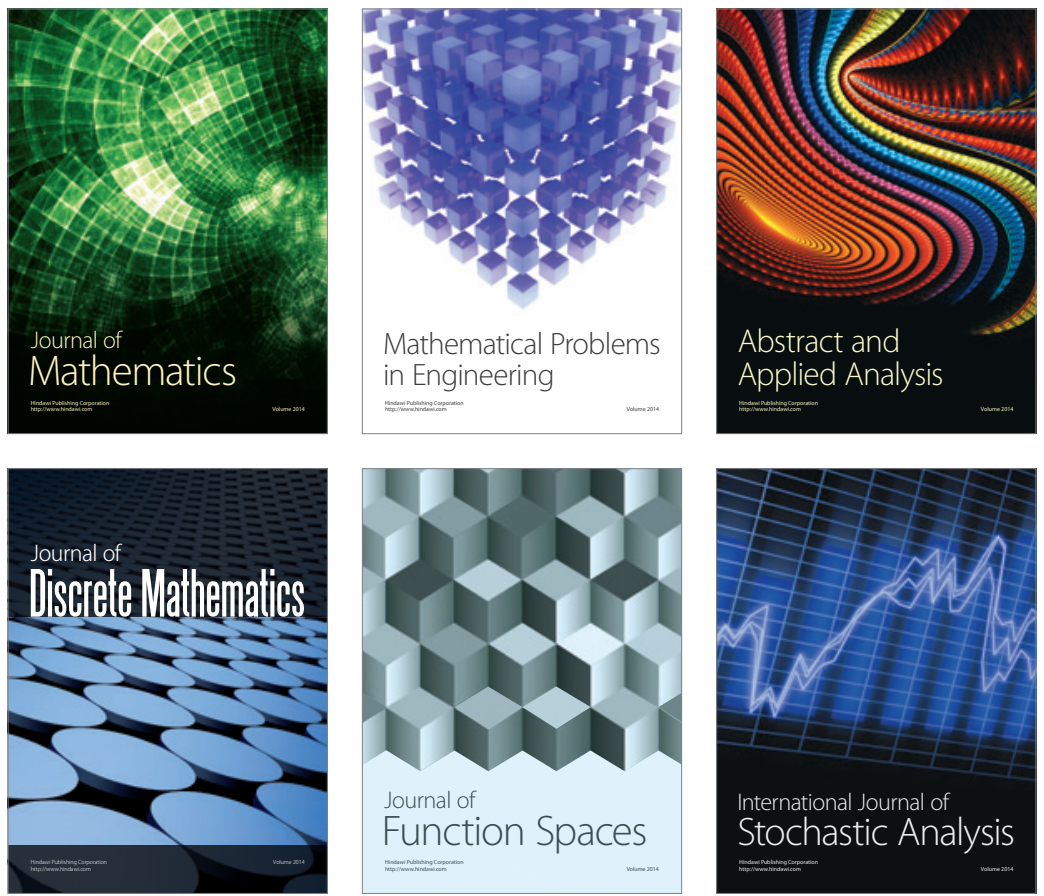

Journal of

Function Spaces

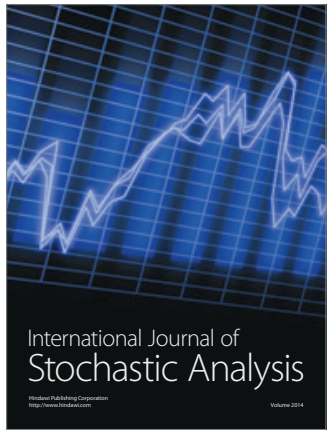

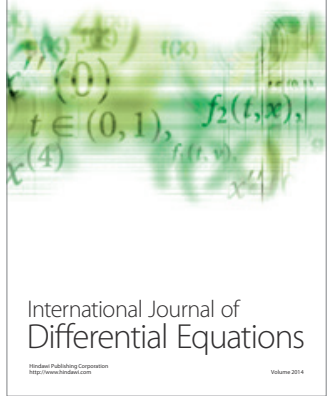
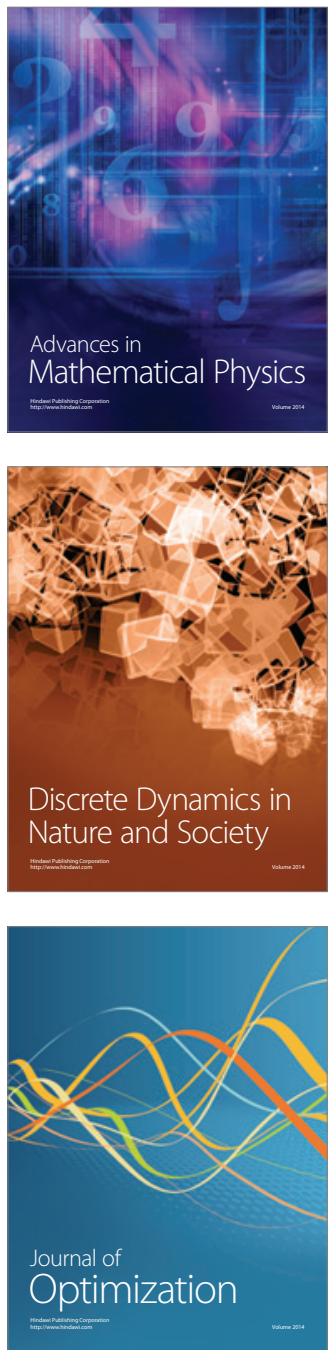